\title{
Avaliação da Percepção de Graduandos da USP Referente à Integração do Ensino Acadêmico com a Tecnologia em Saúde
}

\section{Evaluation of the Perception of USP Undergraduate Students Regarding the Integration of Academic Education with Health Technologies}

Mariane Helen de Oliveira*1, Larissa Novais da Silva Lopes ${ }^{1}$, Luiza Lujan São João ${ }^{1}$, Ingrid Brandimiller Gottsfritz ${ }^{1}$, Giovanna Bifulco Sproesser ${ }^{1}$, Ismael Alves Lucena Gomes², Tamiris Isabeli da Silva ${ }^{1}$, Débora dos Santos Pereira ${ }^{1}$, Wolney Lisboa Conde ${ }^{1}$

${ }^{1}$ Departamento de Nutrição da Faculdade de Saúde Pública da Universidade de São Paulo.

Escola de Enfermagem da Universidade de São Paulo.

* Autora para correspondência: marianehelen@usp.br

\begin{abstract}
RESUMO
A demanda em integrar ensino acadêmico e as Tecnologias em Informação e Comunicação (TICs) é crescente nos dias atuais, visto que os avanços na tecnologia impactam diretamente a produção científica e estão fortemente presentes no cotidiano dos universitários. $\mathrm{O}$ objetivo deste trabalho foi avaliar a percepção de graduandos da área da saúde da Universidade de São Paulo, campus capital, com relação à integração entre ensino acadêmico e tecnologia, por meio de um questionário eletrônico. O questionário apresentava questões sobre o perfil do graduando, o acesso a plataformas digitais durante a formação acadêmica e na prática clínica e os conhecimentos em tecnologias adquiridos durante o curso. As frequências absoluta e relativa referentes a sexo, curso, acesso a dispositivos móveis e percepção dos universitários foram calculadas. O questionário obteve um total de 57 participantes, sendo 46 (80,7\%) do sexo feminino e 11 (19,3\%) do sexo masculino. Dentre os participantes, 93\% referiram o uso de plataformas digitais na formação acadêmica, 94,7\% demonstraram interesse em aprender mais sobre tecnologia durante a graduação, e mais de $90 \%$ apontaram que as ferramentas tecnológicas podem auxiliar na compreensão dos conteúdos ministrados em sala de aula e na aproximação da prática profissional. Evidências apontam a necessidade de implementação das TICs no sistema universitário, assim como o estímulo para a incorporação de ferramentas tecnológicas pelos docentes. Além disso, é preciso construir estratégias de ensino-aprendizagem com recursos tecnológicos que acolham o conteúdo necessário para a formação do profissional da saúde.
\end{abstract}

Palavras-Chave: Práticas Interdisciplinares; Educação Superior; Ciências da Saúde; Tecnologia; Aplicativos Móveis.

\begin{abstract}
Nowadays, the demand for integrating Information and Communication Technologies (ICTs) in higher education has been increasing. Also, the advances in technology have direct impact on scientific production and they are present in the lives of the undergraduate students. The objective of this study was to evaluate the perception about the integration between technology and education of undergraduate students of the health area courses from the University of São Paulo, campus capital, through an electronic questionnaire. The questionnaire presented questions about the undergraduate student profile, access to digital platforms during their course and in the clinical practice, and knowledge of technologies acquired during the undergraduate course. The absolute and relative frequencies regarding sex, major, access to mobile devices and perception of undergraduate students were calculated. The questionnaire obtained a total of 57 participants, being 46 (80.7\%) women and $11(19.3 \%)$ men. From them, 93\% of the students reported using digital platforms in undergraduate courses, $94.7 \%$ expressed interest in learning more about technology during college, and over $90 \%$ pointed out that technological tools can help in to understand the contents in classes and to approximate them to professional practice. Studies show a need to implement ICTs in the university system, as well as stimulation for the incorporation of technological tools by the teachers. In addition, it is necessary to improve teaching-learning strategies with technological resources that integrate the appropriate content for the training of health professionals.
\end{abstract}

Keywords: Interdisciplinary Placement; Higher Education; Health Sciences; Technology; Mobile Applications. 


\section{Introdução}

Compreender a articulação entre a atuação profissional e os conteúdos de disciplinas é um grande desafio durante a formação acadêmica, principalmente com os avanços tecnológicos, que impactam diretamente na produção científica. Segundo Pessoni e Goulart (2015), a maior parte dos estudantes que ingressam no ensino superior possuem acesso a diversas tecnologias como smartphomes, computadores, rede de internet, mídias sociais, entre outros.

$\mathrm{Na}$ área da saúde, as tecnologias que unem conhecimento técnico e científico contribuem para o diagnóstico de pacientes, prevenção de doenças e definição de possíveis tratamentos, sendo, portanto, ferramentas importantes para o profissional da área de saúde durante a sua formação acadêmica (SANTOS et al., 2016).

Ainda de acordo com Pessoni e Goulart (2015), a implantação dessas tecnologias desde a graduação oferece grandes vantagens para os estudantes da área de saúde, que utilizarão essas ferramentas em sua prática profissional, já que essas tecnologias têm sido cada vez mais usadas no mercado de trabalho, através, por exemplo, do registro e armazenamento de dados dos pacientes.

No que concerne ao avanço da tecnologia, em 2016 o Ministério da Saúde publicou uma nova versão da Política Nacional de Informação e Informática em Saúde, a fim de melhorar a qualidade do uso de tecnologias nesta área. Dentre as diretrizes dessa Política, o Ministério alocou, na diretriz de formação de pessoal em informação e informática para a saúde no SUS, a inclusão de conteúdos relacionados com a tecnologia e a informação nos cursos da área de saúde de graduação e pós-graduação.

Considerando as Diretrizes Nacionais Curriculares dos cursos de graduação da área da saúde como Nutrição, Medicina, Odontologia, Enfermagem, entre outros, o aluno deve ter como competência o "domínio em tecnologias de comunicação e informação". Sendo assim, a formação nesses cursos deveria contar com a utilização de TICs como ferramentas para o preparo dos estudantes em sua vida profissional.
Masetto (2003), ao rever a Declaração Mundial sobre Educação Superior no Século XXI: Visão e Ação da Unesco, observou que o documento defende a missão de formar profissionais capacitados e responsáveis. Além disso, coloca em destaque a função ética da universidade, a necessidade de reforçar a cooperação da academia com o mundo do trabalho, analisando e prevenindo as necessidades da sociedade. Ainda segundo a Declaração, algumas mudanças no ensino superior são necessárias, dentre elas a incorporação crítica da tecnologia no ambiente de ensino, uma vez que esses aspectos poderão provocar inovações significativas e relevantes. Com isso, destaca-se a importância da inclusão de disciplinas que envolvam TICs nas grades curriculares, de modo que contribuam para o aperfeiçoamento e a exploração de novos conhecimentos.

Portanto, é necessário avaliar a compreensão e o interesse dos estudantes a respeito do uso de ferramentas tecnológicas durante a graduação ou na prática clínica, a fim de analisar a integração entre a tecnologia e o ensino acadêmico.

\section{Objetivo}

Avaliar a percepção de graduandos da área da saúde com relação à integração de ensino acadêmico e tecnologia, por meio de um questionário eletrônico.

\section{Métodos}

Para a avaliação da percepção dos graduandos sobre a integração entre tecnologia e ensino, foi elaborado um questionário com dez perguntas, através da plataforma on-line do Formulários Google. $\mathrm{O}$ questionário estava estruturado em quatro questões objetivas sobre o perfil do graduando e seis questões em escala de Likert a respeito do acesso do estudante a plataformas digitais durante a formação acadêmica e na prática clínica e dos conhecimentos em tecnologias adquiridos durante o curso. As questões estão apresentadas no Anexo.

O questionário foi enviado eletronicamente para alunos dos cursos de graduação em 
Nutrição, Saúde Pública, Enfermagem, Medicina, Fisioterapia, Terapia Ocupacional, Psicologia e Odontologia da Universidade de São Paulo, campus capital.

As frequências absoluta e relativa referentes a sexo, curso e acesso a dispositivos móveis foram calculadas. O nível de aprendizado em tecnologia na área da saúde e a importância do uso de ferramentas digitais durante a formação acadêmica foram categorizados de acordo com o autorrelato dos estudantes. Além disso, foi avaliada e calculada, através da frequência relativa, a opinião dos estudantes com relação ao uso de plataformas digitais durante a formação acadêmica, durante a prática clínica, e sobre o uso de tecnologia para acelerar o processo de atendimento nas unidades básicas de saúde (UBS). Todas as análises estatísticas foram realizadas no software $R$ versão 3.6 para Windows.

\section{Resultados e Discussão}

O questionário aplicado obteve um total de 57 participantes, sendo 46 (80,7\%) do sexo feminino e $11(19,3 \%)$ do sexo masculino. Todos os estudantes relataram possuir pelo menos um dispositivo móvel com acesso à internet, e 93\% referiram seu uso em auxílio na formação acadêmica. A distribuição dos participantes com relação ao curso de origem está apresentada na tabela 1 . Nota-se que a maior parte dos estudantes que responderam ao questionário era do curso de Nutrição, enquanto que o curso de Fisioterapia só contou com um participante.

Com relação ao curso atual, os estudantes relataram baixo/médio nível de aprendizagem em tecnologias voltadas para a área da saúde e evidenciaram a importância do uso das ferramentas tecnológicas durante a formação acadêmica. Os resultados podem ser observados nas figuras 1 e 2 .

Dos estudantes entrevistados, $94,7 \%$ demonstraram interesse em aprender mais sobre tecnologia durante a graduação, mais de $90 \%$ apontou que as ferramentas tecnológicas podem auxiliar na compreensão dos conteúdos ministrados em sala de aula e na aproximação da prática profissional.
E com relação à atuação clínica, 89,5\% das respostas consideram viável o uso de aplicativos móveis por profissionais de saúde para otimizar os atendimentos.

De acordo com Amem e Nunes (2006), esse novo modelo de comunicação com múltiplas fontes de informação por meio do uso das mais diversas tecnologias demanda uma nova forma de aprendizagem que vá além da sala de aula. Para isso, é necessário que os estudantes se apropriem dos conhecimentos técnicos e científicos integrados aos meios de tecnologia e comunicação. Além de favorecer a prática clínica também integrada aos meios tecnológicos, esse tipo de ensino nos permite compreender que estamos cada vez mais conectados e interdependentes.

No entanto, geralmente ainda é dada maior importância ao conteúdo técnico do que a toda essa rede de contribuições que convergem em um certo conhecimento acerca desse conteúdo. Sendo assim, faz-se necessária a adoção de práticas de ensino e aprendizagem que integrem a teoria à prática, a fim de formar profissionais flexíveis, independentes, inovadores e que saibam construir conhecimentos em conjunto com outros (GADELHA et al., 2019).

Apoiando-se nos estágios do ciclo de aprendizagem com o uso de TICs proposto por Mayes e Fowler (1999), Sugrue (2000) aponta papéis da estratégia no processo educativo que são interdependentes, tais quais: acesso e organização da informação; atividades autênticas, sendo a aplicação de forma prática de teorias, com o intuito de potencializar os processos de aprendizagem; aprendizagem colaborativa, relacionada à comunicação, ao diálogo e à interação entre docente e discente.

Barbosa (2016) destaca que, apesar do grande potencial das TICs, somente a sua implantação no ensino não é suficiente para utilizar todo seu potencial de facilitadoras da aprendizagem, pois muitas vezes a inserção dessas tecnologias não acompanha um ensino formador para preparo dos docentes que irão utilizar esse método. Além disso, não há uma análise do conhecimento dos alunos sobre as TICs que serão utilizadas. Por 


\begin{tabular}{|c|c|c|}
\hline & \multicolumn{3}{|c|}{ Estudantes $\mathrm{n}=57$} \\
\hline Eurso & $\mathbf{n}$ & $\mathbf{( \% )}$ \\
\hline Enfermagem & 4 & 7,00 \\
\hline Medicioterapia & 1 & 1,80 \\
\hline Nutrição & 2 & 3,50 \\
\hline Saúde Pública & 46 & 80,70 \\
\hline
\end{tabular}

Tabela 1 - Garacterização dos participantes com relação aos cursos em que estão matriculados na Universidade de São Paulo, campus capital, 2018. n = frequência absoluta, $\%=$ frequência relativa.

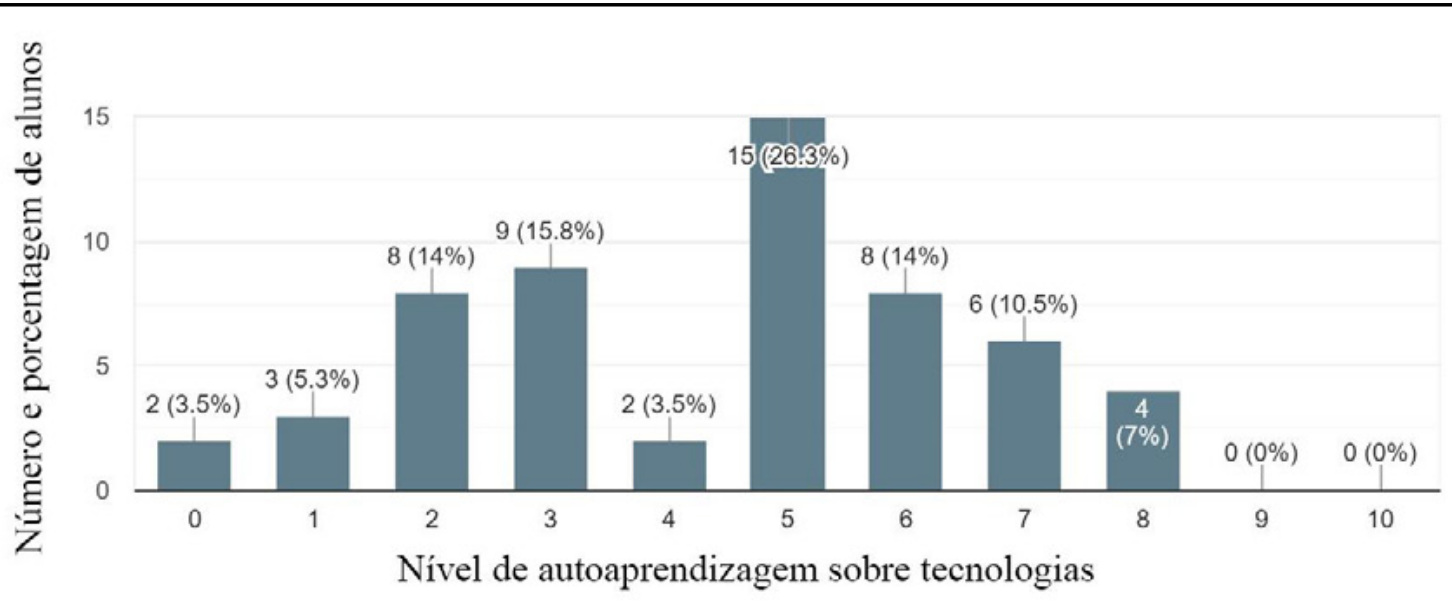

Figura 1 - Autoclassificação do nível de aprendizagem sobre tecnologias na área da saúde relatado pelos estudantes entrevistados neste estudo em 2019.

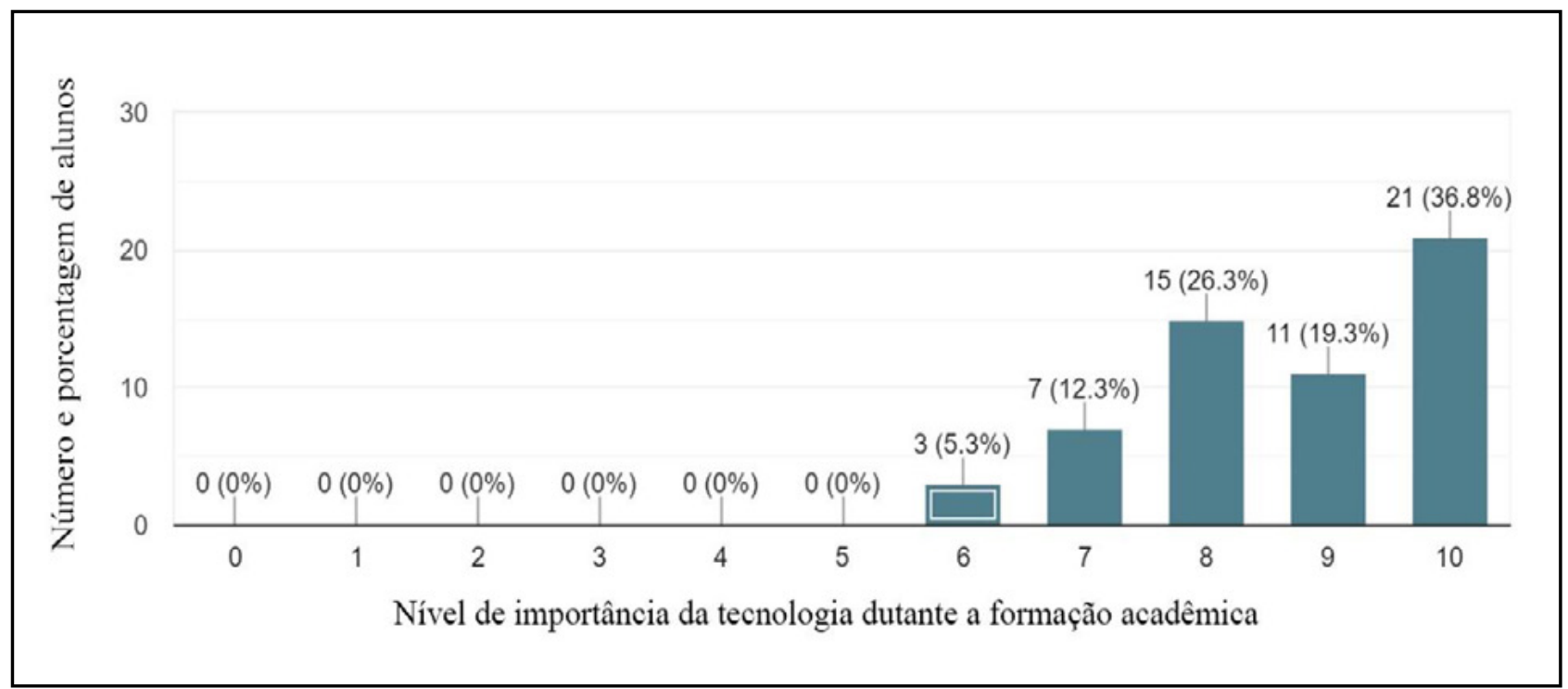

Figura 2 - Autoclassificação do nível de importância da tecnologia durante a formação acadêmica relatada pelos estudantes entrevistados neste estudo em 2019. 
isso, é necessário que o corpo docente seja capacitado para usar as tecnologias de forma eficaz, facilitando a aprendizagem e integrando o ensino.

Ainda segundo Cavalcante e Vasconcellos (2007), pontos críticos impedem a ampliação da tecnologia no ambiente acadêmico da área de saúde, são eles: resistência de professores e pesquisadores a aderir às novas tecnologias, dificuldade de aquisição e investimento de tempo e esforço para adaptação a elas.

Uma pesquisa quantitativa realizada por Ramos et al. (2018) com coordenadores de cursos da área da saúde, professores supervisores e coordenadores de estágios curriculares obrigatórios da Universidade Federal de Pernambuco (UFPE) demonstrou que, dos 27 entrevistados, nenhum afirmou possuir alto domínio das TICs e apenas $18,5 \%$ responderam ter formação para o uso dessas tecnologias.

A tecnologia de informação em saúde encontra-se em uma crescente implantação nos setores de atendimento à saúde, culminando para que as instituições de ensino reconheçam a importância da informática no treinamento primário de profissionais da saúde (BARBOSA, 2015). Nos últimos anos, a inserção das TICs tornou-se um ponto presente nas Diretrizes Curriculares de diversos cursos de graduação, especialmente nos cursos da área da saúde (MORAIS et al., 2014). Segundo estudo de Haddad et al. (2018), foi incluída a diretriz da importância do uso de TICs para a graduação na reorientação curricular do curso de Odontologia da Faculdade de Odontologia da USP (FOUSP).

É evidente o espaço de oportunidade para estimular os alunos a trabalharem com este espectro na educação. Não somente a tecnologia em si é importante, pelo fato de ser uma ferramenta interativa e lúdica, mas também o questionamento educacional que o docente propõe ao adotar uma inovação, fazendo com que o aluno se torne participante ativo na construção de seu aprendizado.

\section{Considerações Finais}

Globalmente, as tendências apontam que as tecnologias baseadas na infraestrutura da internet têm se destacado no ensino superior, proporcionando novas formas de comunicação e interação entre os membros da comunidade acadêmica.

Observa-se que os graduandos fazem uso frequente de plataformas digitais e consideram que estas facilitam o aprendizado, assim como a prática profissional. Além disso, os estudantes manifestam interesse em aperfeiçoar seus conhecimentos através do uso contínuo de ferramentas tecnológicas durante o curso, uma vez que essas plataformas permitem a atualização progressiva. Porém, ainda há certa resistência por parte dos docentes, que alegam dificuldade de aquisição das diversas tecnologias e necessidade de um grande investimento de tempo e esforço para se adaptarem a elas, visto que não há amplo domínio sobre suas funcionalidades entre professores e pesquisadores.

É preciso construir estratégias de ensino-aprendizagem com recursos tecnológicos que acolham o conteúdo necessário para a formação do profissional da saúde. Sendo assim, este é um material interessante para incentivar novas pesquisas na área, bem como ações e estratégias pedagógicas para implementar um maior uso da tecnologia no ensino superior.

\section{Referências Bibliográficas}

AMEM, Bernadete Malmegrim Vanzella; NUNES, L. C. "Tecnologias de Informação e Comunicação: Contribuições para o Processo Interdisciplinar no Ensino Superior". Rev. Bras. Educ. Med., Rio de Janeiro, vol. 30, n. 3, dez. 2006, pp. 171-180. Disponível em: $<$ http://www.scielo.br/scielo.php?script=sci_arttext\&pid=S010055022006000300008\&lang=pt $>$.

BARBOSA, Alexandre. TIC Saúde 2013: Pesquisa sobre o Uso das Tecnologias de Informação e Comunicasãa nos Estabelecimentos de Saúde Brasileiros. São Paulo: Comitê Gestor da Internet no Brasil, 2015. [livro eletrônico] Disponível em: <https://cetic.br/media/docs/publicacoes/2/tic-saude-2013.pdP.

BARBOSA, Tatiana Péret. "Tecnologias Digitais: Desafios e Perspectivas no Ensino Superior em Saúde". Percurso Acadêmico, Belo Horizonte, vol. 6, n. 12, jul./ dez. 2016.

BRASIL. Ministério da Saúde. Politica Nacional de Informação e Informática em Saúde. Brasília: Ministério da Saúde, 2016 p. 56.

BRASIL. "Resolução CNE/CES 1133, de 3 de outubro de 2001". Institui Diretrizes Curriculares 
Nacionais dos Cursos de Graduação em Enfermagem, Medicina e Nutrição. Diário Oficial da União, Brasília, DF, 7 out. 2001. Seção 1E, p. 131.

BRASIL. "Resolução GNE/CES n. 5, de 7 de novembro de 2001". Institui Diretrizes Curriculares Nacionais do Gurso de Graduação em Nutrição. Diário Oficial da União, Brasília, DF, 9 nov. 2001. Seção 1, p. 39.

CAVALCANTE, Maria Tereza Leal \& VASCONCELLOS, M. M. "Tecnologia de Informação para a Educação na Saúde: Duas Revisões e uma Proposta". Ciênc. Saúde coletiva, Rio de Janeiro, vol. 12, n .3, maio/jun. 2007. Disponível em: http:// www.scielo.br/scielo.php?script $=$ sci_arttext\&pi$\mathrm{d}=\mathrm{S} 141432832019000100253 \&$ lang $=\mathrm{pt}$.

GADELHA, Marília Moreira Torres.; ANDRADE, M. E. de.; SILVA, J. M. A.; BEZERRA, I. C. B.; CARMO, A.P. do\&FERNANDES, M. C. "Tecnologias Educativas no Processo Formativo: o Discurso dos Acadêmicos de Enfermagem". Rev. Enferm UFPE On-line, Recife, vol. 13, n. 1, jan. 2019, pp. 155-161.

HADDAD, Ana Estela; ROMERO, J. S. L.; ALENCAR, C. J. F.; RAGGIO, D. P.; CIAMPONI, A. L. \& BONEGKER, M. "A Política Nacional de Educação Superior na Saúde e a Reorientação Curricular da FOUSP: Análise Comparativa do Desempenho e Percepção dos Estudantes". Revista de Graduação USP, vol. 3, n. 2, jul. 2018.

MASETTO, Marcos. "Inovação na Educação Superior". Interface - Comunic., Saúde, Educ., vol. 8, n. 14, set. 2003-fev. 2004 .

MAYES, J. Terry \& FOWLER, C. J. H. Learning Technology and Usability: a Framework for Understanding Courseware. Interacting with Computers, vol. 11, n. 5, 1999, pp. 485-497.

MORAIS, Nídia Salomé; POMBO, L.; BATISTA, J.; MOREIRA, A. \& RAMOS, F. "Uma Revisão de Literatura sobre o Uso das Tecnologias de Comunicação no Ensino Superior". PRISMA.COM, 2014, 24, pp. 162-185.

PESSONI, Arquimedes \& GOULART, E. "Tecnologias e o Ensino na Área da Saúde". ABCS Health Sciences, vol. 40, n. 3, 2015, pp. 270-275.

RAMOS, Emille Raiza Luna Gomes; SOUZA, F. B. de. \& CABRAL DE MELO, M. M. D. "Incorporação das Tecnologias de Informação e Comunicação na Integração Ensino-Serviço dos Cursos de Saúde de uma Universidade Pública". Revista da Abeno, Pernambuco, vol. 18, n. 3, 2018, pp. 159-168.

SANTOS, Zélia Maria de Sousa Araújo; FROTA, M. A. \& MARTINS, A. B. T. Tecnologias em Saúde: da Abordagem Teórica à Construção e Aplicação no Cenário do Cuidado. Fortaleza: EdUECE, 2016. p. 482

SUGRUE, B. "Cognitive Approaches to WebBased Instruction". In: LAJOIE, S. (ed.). Computers as Cognitive Tools: No More Walls. Mahwah, New Jersey: Lawrence Erlbaum Associates, 2000, pp. 133-162.

Publicado em 28/07/2020. 


\section{ANEXO}

1) Sexo:

( ) Feminino ( ) Masculino ( ) Prefiro não informar

2) Curso:

( ) Nutrição ( ) Saúde Pública ( ) Enfermagem ( ) Medicina ( ) Fisioterapia

( ) Terapia Ocupacional ( ) Psicologia ( ) Odontologia ( ) Outro

3) Você possui ao menos um dispositivo móvel (celular) com acesso à internet?

( ) $\operatorname{Sim}($ ) Não

4) Você utiliza plataformas digitais e aplicativos para auxiliar na sua formação acadêmica?

( ) $\operatorname{Sim}($ ) Não

5) Na sua visão, o aumento de ferramentas tecnológicas facilita a compreensão do conteúdo apresentado nas aulas e aproxima os estudantes da prática profissional?

( ) Discordo totalmente ( ) Discordo parcialmente ( ) Indiferente

( ) Concordo parcialmente ( ) Concordo totalmente

6) De zero a dez (0-10), o quão importante é a tecnologia para você como estudante?

( ) 0 ( ) 1 ( ) 2 ( ) 3 ( ) 4 ( ) 5 ( ) 6 ( ) 7 ( ) 8 ( ) 9 ( ) 10

7) Você acha viável o uso de dispositivos móveis na prática clínica de um profissional de saúde para otimizar o atendimento?

( ) Discordo totalmente ( ) Discordo parcialmente ( ) Indiferente

( ) Concordo parcialmente ( ) Concordo totalmente

8) De zero a dez (0-10), o quanto você aprendeu sobre tecnologias voltadas para a área da saúde durante a graduação?

( ) 0 ( ) 1 ( ) 2 ( ) 3 ( ) 4 ( ) 5 ( ) 6 ( ) 7 ( ) 8 ( ) 9 ( ) 10

9) Você tem interesse em aprender mais sobre tecnologia durante a graduação?

( ) Discordo totalmente ( ) Discordo parcialmente ( ) Indiferente

( ) Concordo parcialmente ( ) Concordo totalmente

10) Como estudante na área da saúde, você acredita que a tecnologia pode contribuir para acelerar o processo de atendimento nas Unidades Básicas de Saúde (UBS) da rede pública de serviços de saúde?

( ) Discordo totalmente ( ) Discordo parcialmente ( ) Indiferente

( ) Concordo parcialmente ( ) Concordo totalmente 